\title{
Subthalamic deep brain stimulation improves sleep and excessive sweating in Parkinson's disease
}

\author{
Silje Bjerknes $\mathbb{D}^{1,2 凶}$, Inger Marie Skogseid $\mathbb{D}^{1}$, Tuva Jin Hauge $\mathbb{D}^{2}$, Espen Dietrichs $\mathbb{D}^{1,2}$ and Mathias Toft $\mathbb{D}^{1,2}$
}

Parkinson's disease (PD) is a complex multisystem disorder with motor and non-motor symptoms (NMS). NMS may have an even greater impact on quality of life than motor symptoms. Subthalamic nucleus deep brain stimulation (STN-DBS) has been shown to improve motor fluctuations and quality of life, whereas the effects on different NMS have been less examined. Sleep disturbances and autonomic dysfunction are among the most prevalent NMS. We here report the efficacy of STN-DBS on sleep disturbances and autonomic dysfunction. In the parent trial, 60 patients were included in a single-center randomized prospective study, with MDSUPDRS III and PDQ-39 as primary endpoints at 12 months of STN-DBS. Preplanned assessments at baseline and postoperatively at 3 and 12 months also included Parkinson's Disease Sleep Scale (PDSS); Scopa-Aut; and MDS-UPDRS I, II, and IV. We found that STNDBS had a significant and lasting positive effect on overall sleep quality, nocturnal motor symptoms and restlessness, and daytime dozing. Several aspects of autonomic dysfunction were also improved at 3 months postoperatively, although at 12 months only thermoregulation (sudomotor symptoms) remained significantly improved. We could not identify preoperative factors that predicted improvement in PDSS or Scopa-Aut. There was a close relationship between improved autonomic symptoms and improved quality of life after 1 year. NMS and especially sleep and autonomic dysfunction deserve more focus to improve patient outcomes further.

npj Parkinson's Disease (2020)6:29; https://doi.org/10.1038/s41531-020-00131-0

Parkinson's disease (PD) is a common neurodegenerative disease diagnosed by the classical motor signs of bradykinesia, tremor, and/or rigidity ${ }^{1}$. However, it has been increasingly recognized that PD is a complex multisystem disorder with motor and non-motor symptoms $(\mathrm{NMS})^{2,3}$. NMS can be divided into four domains: autonomic, sleep, neuropsychiatric, and sensory symptoms, including pain ${ }^{4,5}$. NMS have a significant impact on quality of life $^{6}$, often even to a greater extent than motor symptoms ${ }^{7-10}$.

Treatment of PD aims at reducing symptoms, as no treatment that can modify disease progression has been established. Symptom reduction through treatment with levodopa and other dopaminergic agents is highly effective, but in later disease stages the management of motor and non-motor fluctuations can be challenging. When oral medical treatment proves to be insufficient in reducing tremor or motor fluctuations, deep brain stimulation (DBS) is an effective and well-established symptomatic treatment option ${ }^{11-13}$. The significance of NMS in the DBS-treated cohorts and the effect of DBS on these symptoms are reflected by several publications on this topic during the past years ${ }^{14-16}$.

NMS burden has been shown to increase by disease duration, age, and with more severe motor impairment ${ }^{17}$. The patients eligible for subthalamic nucleus (STN)-DBS typically are in an advanced stage of the disease when the NMS burden is high, but the reported effects of STN-DBS on NMS are somewhat conflicting in the literature. Some studies have shown that DBS is better than dopamine replacement therapy in reducing $\mathrm{NMS}^{18}$, others have shown only minor effects of STN-DBS compared to levodopa and that the pattern of NMS was similar in a non-operated PD reference population ${ }^{19,20}$. Improvements in NMS have, however, been shown in several studies ${ }^{21-23}$. The literature thus presents variable findings in this research field, which is impeded by the use of many different methods and questionnaires, including nonvalidated scales or questionnaires ${ }^{24,25}$.
Sleep problems have been reported in 60-98\% of PD patients ${ }^{26,27}$, but autonomic NMS are also common (nocturia in $62 \%$ of patients, urinary urgency in $58 \%$, hypersalivation in $48 \%)^{28}$. Despite the high frequency, such symptoms have been less investigated than the total NMS burden. To our knowledge, few long-term studies have investigated the effect of STN-DBS on sleep and dysautonomia.

In this study, we have evaluated the impact of bilateral STN-DBS on sleep problems and dysautonomia during the first year of chronic stimulation and explored their relationship to motor and quality-of-life outcomes.

\section{RESULTS}

In this study, 60 patients were included, operated, and examined preoperatively, with planned follow-up after 3 and 12 months of continuous STN-DBS ${ }^{29}$. There were no significant differences in Parkinson's Disease Sleep Scale (PDSS) or Scopa-Aut (scales for outcomes in PD-autonomic) between the two randomization groups of the parent study (see "Methods" section), neither regarding improvement from baseline to the 12-month follow-up nor in the scores at the different time points. Thus, for the purpose of this paper, we analyzed the two groups as one sample. Median age at surgery was 62 (44-71) years, disease duration 11 (4-23) years, and median preoperative Movement Disorder Society revision of the Unified Parkinson's Disease Rating Scale (MDSUPDRS) III scores in the medication-off/-on states were $47 / 13$ (range 23-78/1-45). Complete baseline characteristics are presented in Table 1.

Three patients had surgical site infections with subsequent hardware explantation and discontinuation of neurostimulation (before the 3-month follow-up), and two patients were lost to 1 -year follow-up. Significant improvements were found for

\footnotetext{
'Department of Neurology, Oslo University Hospital, Oslo, Norway. ${ }^{2}$ Institute of Clinical Medicine, University of Oslo, Oslo, Norway. ${ }^{凶}$ email: sbbjsk@ous-hf.no
} 
Table 1. Baseline characteristics.

\begin{tabular}{|c|c|}
\hline \multicolumn{2}{|l|}{ Gender $(n(\%))$} \\
\hline Male & $45(75)$ \\
\hline Female & $15(25)$ \\
\hline Age at surgery & $62(44-71)$ \\
\hline Disease duration (years) & $11(4-23)$ \\
\hline \multicolumn{2}{|l|}{ HAD } \\
\hline Anxiety & $4(0-12)$ \\
\hline Depression & $3(0-19)$ \\
\hline Mattis Dementia Rating Scale & $142(131-144)$ \\
\hline LEDD & $1291(428-2490)$ \\
\hline MDS-UPDRS I & $10.5(1-25)$ \\
\hline MDS-UPDRS II & $16.0(0-32)$ \\
\hline \multicolumn{2}{|l|}{ MDS-UPDRS III } \\
\hline Off & $47(23-78)$ \\
\hline On & $13(1-45)$ \\
\hline MDS-UPDRS IV & $10.0(0-16)$ \\
\hline \multicolumn{2}{|l|}{ H\&Y off ( $n(\%))$} \\
\hline 1 & 0 \\
\hline 1.5 & 0 \\
\hline 2 & $20(33)$ \\
\hline 2.5 & $17(28)$ \\
\hline 3 & $10(17)$ \\
\hline 4 & $11(18)$ \\
\hline 5 & $2(3)$ \\
\hline \multicolumn{2}{|l|}{ H\&Y on $(n(\%))$} \\
\hline 1 & $3(5)$ \\
\hline 1.5 & $3(5)$ \\
\hline 2 & $37(62)$ \\
\hline 2.5 & $15(25)$ \\
\hline 3 & $2(3)$ \\
\hline 4 & 0 \\
\hline 5 & 0 \\
\hline
\end{tabular}

Values are medians (min-max). $N=60$ except for HAD $n=58$ and Mattis Dementia Rating Scale $n=50$.

MDS-UPDRS I-IV, Parkinson's Disease Questionnaire-39 (PDQ-39), and levodopa equivalent daily dosage (LEDD) ${ }^{30}$ at both the $3-$ and 12 -month evaluations, as presented in our previous publication ${ }^{29}$ (Table 2).

Changes of PDSS and Scopa-Aut from baseline to 3- and 12-month follow-up

Mean PDSS total score improved significantly from $93.8( \pm 21.3)$ at baseline to $110.0( \pm 21.7)$ at 3 months and $107.3( \pm 21.3)$ at 12 months of STN-DBS. Changes for each domain are shown in Table 2. Overall sleep quality, nocturnal restlessness, nocturnal motor symptoms, and daytime dozing were all significantly improved. For sleep onset and maintenance insomnia, improvements were just above significance level after Bonferroni correction.

The proportion of patients with severe sleep abnormalities (PDSS score $\leq 83$ points) ${ }^{31}$ were reduced from $31 \%$ preoperatively to $14 \%$ at 3 months (McNemar's test, $p=0.001$ ) and to $12 \%$ at 12 months $(p=0.005)$, see Fig. 1 .
Table 2. Changes from preoperative to the 3- and 12-month followup for PDSS, Scopa-Aut, PDQ-39, MDS-UPDRS I-IV, and LEDD.

\begin{tabular}{|c|c|c|c|c|}
\hline & Preoperative & 3 months & 12 months & $p$ \\
\hline PDSS, $n$ & 58 & 49 & 52 & \\
\hline PDSS total & $93.8(21.3)$ & $110.0(21.7)$ & $107.3(21.3)$ & $<0.001^{\mathrm{b}}$ \\
\hline $\begin{array}{l}\text { Overall quality of } \\
\text { night's sleep (Q1) }\end{array}$ & $5.0(2.8)$ & $6.5(2.9)$ & $6.2(2.8)$ & $<0.001^{b}$ \\
\hline $\begin{array}{l}\text { Sleep onset and } \\
\text { maintenance insomnie } \\
(\mathrm{Q} 2, \mathrm{Q} 3)\end{array}$ & $10.9(4.1)$ & $12.9(4.8)$ & $12.0(4.4)$ & 0.007 \\
\hline $\begin{array}{l}\text { Nocturnal } \\
\text { restlessness (Q4, Q5) }\end{array}$ & $11.5(5.2)$ & $14.1(4.9)$ & $13.0(5.9)$ & $0.003^{b}$ \\
\hline $\begin{array}{l}\text { Nocturnal } \\
\text { psychosis }(\mathrm{Q} 6, \mathrm{Q} 7)\end{array}$ & $16.8(3.4)$ & $17.5(2.7)$ & $16.4(4.9)$ & 0.120 \\
\hline Nocturia $(\mathrm{Q} 8, \mathrm{Q9})$ & $11.7(3.8)$ & $12.4(4.2)$ & $12.5(4.0)$ & 0.055 \\
\hline $\begin{array}{l}\text { Nocturnal motor } \\
\text { symptoms (Q10-Q13) }\end{array}$ & $26.2(8.9)$ & $32.4(6.0)$ & $33.1(6.4)$ & $<0.001^{b}$ \\
\hline $\begin{array}{l}\text { Sleep } \\
\text { refreshment (Q14) }\end{array}$ & $5.2(3.0)$ & $6.3(3.0)$ & $6.0(3.1)$ & 0.041 \\
\hline $\begin{array}{l}\text { Daytime } \\
\text { dozing (Q15) }\end{array}$ & $6.5(3.3)$ & $8.0(2.4)$ & $7.6(3.2)$ & $<0.001^{b}$ \\
\hline Scopa-Aut, $n$ & 58 & 54 & 53 & \\
\hline Scopa-Aut total & $16.9(8.2)$ & $13.0(6.8)$ & $15.2(8.4)$ & $<0.001^{b}$ \\
\hline $\begin{array}{l}\text { Gastrointestinal } \\
\text { (Q1-7) }\end{array}$ & $5.0(3.3)$ & $4.2(2.9)$ & $4.8(3.6)$ & 0.059 \\
\hline Urinary (Q8-13) & $5.8(3.7)$ & $4.4(2.7)$ & $5.2(3.7)$ & 0.054 \\
\hline $\begin{array}{l}\text { Cardiovascular } \\
\text { (Q14-16) }\end{array}$ & $0.8(1.1)$ & $0.6(0.9)$ & $0.6(1.0)$ & 0.172 \\
\hline $\begin{array}{l}\text { Thermoregulatory } \\
\text { (Q17-21) }\end{array}$ & $3.2(2.5)$ & $1.8(1.8)$ & $2.2(2.3)$ & $<0.001^{b}$ \\
\hline Pupillomotor (Q19) & $0.7(0.8)$ & $0.5(0.6)$ & $0.7(0.9)$ & 0.059 \\
\hline Sexual (Q22-25) & $1.4(1.8)$ & $1.5(1.8)$ & $1.6(2.0)$ & 0.622 \\
\hline PDQ, $n$ & 59 & 51 & 53 & \\
\hline PDQ-39 & $27(12)$ & $18(13)$ & $20(15)$ & $<0.001$ \\
\hline MDS-UPDRS, $n$ & 60 & 56 & 55 & \\
\hline MDS-UPDRS I & $11.3(6.1)$ & $8.7(5.8)$ & $9.0(5.7)$ & $<0.001$ \\
\hline MDS-UPDRS II & $16.8(7.3)$ & $10.9(6.5)$ & $11.5(6.7)$ & $<0.001$ \\
\hline MDS-UPDRS III off & $49(13)$ & $19(10)$ & $20(9)$ & $<0.001$ \\
\hline MDS-UPDRS III on & $14(9)$ & $12(7)$ & $12(7)$ & $<0.001$ \\
\hline MDS-UPDRS IV & $9.6(3.5)$ & $2.5(3.0)$ & $2.6(3.7)$ & $<0.001$ \\
\hline LEDD & 1301 (441) & 689 (373) & 639 (328) & $<0.001$ \\
\hline
\end{tabular}

Mean (SD) are shown for all patients who had complete scores at each time point.

Significance of changes ( $p$ value presented) was tested with one-way repeated-measures ANOVA in subjects with values at all three time points; for PDSS $n=44$ and for Scopa-Aut $n=51$.

${ }^{\text {a}}$ Friedman test.

${ }^{\mathrm{b}}$ Significant also after Bonferroni correction (for PDSS significance level $p<0.006$, for Scopa-Aut significance level $p<0.007$ ).

Scopa-Aut total score improved significantly overall over the three time points (repeated-measures analysis of variance (ANOVA)), see Table 2. Preoperative mean $( \pm S D)$ score was reduced from $16.9( \pm 8.2)$ to $13.0( \pm 6.8)$ at 3 months of STN-DBS (pairwise comparisons, $p<0.001)$. At 12 months, it was $15.2( \pm 8.4)$ $(p=0.073)$. Analyses of each Scopa-Aut domain showed significant improvement at 12 months only for the thermoregulatory domain. Interestingly, from that domain, only excessive sweating during day and night improved significantly (sudomotor symptoms, question $17(p=0.001)$ and question $18(p=0.044)$. 


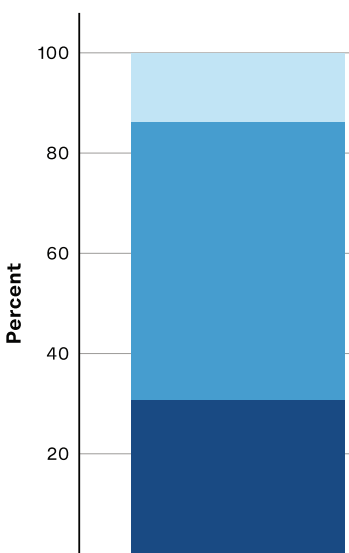

Preoperatively

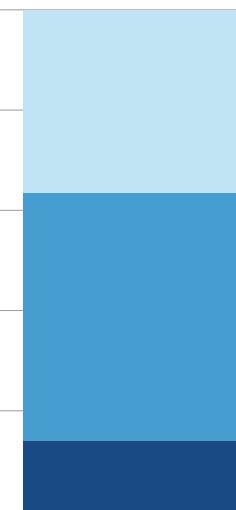

3 months

Time

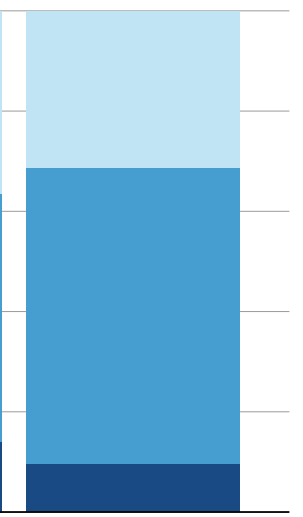

12 months

PDSS $\leq 83$

Fig. 1 PDSS severity distribution. Columns show the percentage of patients with PDSS $\leq 83$ (indicating severe sleep abnormality), PDSS $84-120$ (indicating sleep disturbances), and PDSS $\geq 121$ (indicating no sleep disturbances), preoperatively $(n=58)$ versus after $3(n=49)$ and 12 months $(n=51)$ of STN-DBS. McNemar's test preoperative to 3 months $(p=0.001)$ and preoperative to 12 months $(p=0.005)$.

Table 3. Multivariable linear regression analyses.

1. Prediction model of PDSS difference (from preop. to 12 months)

Preoperative

Scopa-Aut

PDQ-39

MDS-UPDRS II

MDS-UPDRS III off

LEDD

Model fit:

$R$ square adjusted

( $p$ value)

$1 \%$

(0.468)
2. Covariance model of PDSS difference (from preop. to 12 months)

Difference from preoperative to 12 months

B-coefficient (S.E.)

$0.471(0.381)$

Scopa-Aut

$-0.893(0.481)$

$-0.521(0.325)$

PDQ-39

$-0.213(0.312)$

$0.331(0.554)$

MDS-UPDRS ॥

$-0.574(0.456)$

$-0.237(0.238)$

MDS-UPDRS III off

$-0.095(0.245)$

$-0.237(0.238) \quad$ LEDD

$-0.005(0.007)$

$8 \%$

(0.135)

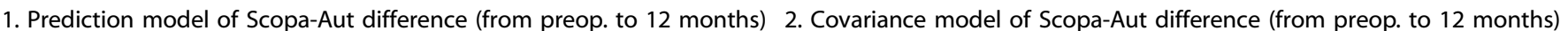

\begin{tabular}{|c|c|c|c|}
\hline Preoperative & $B$-coefficient (S.E.) & Difference from preoperative to 12 months & B-coefficient (S.E.) \\
\hline PDQ-39 & $0.043(0.121)$ & PDQ-39 & $0.308(0.085)^{a}$ \\
\hline MDS-UPDRS ॥ & $0.048(0.170)$ & MDS-UPDRS ॥ & $-0.132(0.154)$ \\
\hline LEDD & $0.003(0.002)$ & LEDD & $0.002(0.002)$ \\
\hline \multicolumn{4}{|l|}{ Model fit: } \\
\hline ( $p$ value) & $(0.590)$ & & $(<0.001)^{\mathrm{a}}$ \\
\hline
\end{tabular}

Dependent variables are the differences in Parkinson's Disease Sleep Scale (PDSS) and Scopa-Aut total scores from preoperative to 12 months of STN-DBS. As independent variables, preoperative scores are used in the prediction models (1, left column) and differences in scores from preoperative to 12 months in the covariance models (2, right column).

PDQ-39 Parkinson's Disease Questionnaire-39, LEDD Levodopa equivalent daily doses, MDS-UPDRS Movement Disorder Society revision of the Unified Parkinson's Disease Rating Scale.

${ }^{\mathrm{a} S i g n i f i c a n t}(p<0.05)$.

Regression analyses

Multivariable linear regression analyses were performed to identify predictive factors and covariables of the changes observed in PDSS and Scopa-Aut scores from baseline to 12 months of STN-DBS. Variables entered into the regression models were chosen based on clinical experience and exploratory correlation analyses. The results are shown in Table 3. For PDSS difference after 1 year of STN-DBS, the predictive model showed goodness of fit of only $1 \%(p=0.468)$ and the model of covariance showed goodness of fit of $8 \%(p=0.135)$. For Scopa-Aut 
difference, the predictive model showed goodness of fit of $3 \%$ $(p=0.590)$, whereas the covariance model showed goodness of fit of $31 \%(p<0.001)$, with improvement in PDQ-39 as the only significant contributor. There is no violation of the assumption of normality, linearity, or multiple collinearity in the models. There are some overlapping questions in the questionnaires that might explain at least parts of the prediction of the variance, but ScopaAut and PDQ-39 only share the questions of feeling unpleasantly hot or cold.

\section{DISCUSSION}

In this prospective study of 60 PD patients treated with STN-DBS, our main finding was improvement of sleep disturbances at 12 months, as evaluated by PDSS. The domains overall sleep quality, nocturnal restlessness, nocturnal motor symptoms, and daytime dozing all improved. Statistically significant improvement in autonomic symptoms was also found at 3 months but was no longer evident at 12 months, except for sudomotor function.

Objective improvement in sleep quality following STN-DBS has been shown by polysomnography in small numbers of patients ${ }^{32-34}$, but there are conflicting results in the literature. Polysomnography cannot detect excessive daytime sleepiness and it is not feasible to study all PD patients with this modality. Therefore, several self-rating questionnaires that solely evaluate sleep have been developed, such as Epworth sleepiness scale, Pittsburgh Sleep Quality Index, and PDSS. Compound scales used to evaluate NMS also include sleep items (UPDRS I and II, NMSS, NMSQ). However, varying use of these scales has resulted in no uniform presentation of sleep symptoms. We have used the PDSS recommended by the MDS Task Force because it includes most of the potential sleep disturbances that PD patients may encounter ${ }^{35}$.

Overall, previous reports indicate that STN-DBS may improve nocturnal sleep in PD patients, particularly sleep quality ${ }^{16}$. However, many previous studies have included small numbers of patients followed for a short time period. In 10 patients with 3 months of follow-up, PDSS showed improved sleep quality but, unlike our findings, without improvement of excessive daytime sleepiness $^{36}$. In two other studies using PDSS, one showed improved scores for daytime sleepiness, sleep quality, and restless legs syndrome $(n=17)$ at 6 months postoperatively ${ }^{37}$, and the other showed significant improvements in PDSS after 6 months $(n=40)$ but not after 12 months $(n=26)^{38}$.

Reduction of LEDD is hypothesized to play a role in the improvement of sleep symptoms, as studies have shown poorer sleep quality and less rapid eye movement (REM) sleep on higher doses of dopaminergic medication taken prior to sleep ${ }^{39,40}$. However, findings in a study that compared untreated PD patients, advanced PD patients (Hoehn \& Yahr (H\&Y) 4-5), and healthy controls indicated that sleep disruption occurs even before pharmacologic therapy and is likely a symptom of the underlying disease as well ${ }^{41}$. In a study comparing globus pallidus interna- and STN-DBS, there was no difference in PDSS improvements, despite a more pronounced LEDD reduction in the STN group. This may imply that the DBS effect on sleep surpasses the effect of dopaminergic medication reduction ${ }^{42}$.

Improvement in nocturnal mobility has also been suggested to be a significant contributor to improved sleep. However, Monaca et al. found only a mild motor improvement, but a significant improvement in sleep. They suggested that motor improvement alone could not fully explain sleep improvement, indicating that a possible direct effect on sleep/wakefulness regulatory centers might be involved ${ }^{34}$. A polysomnography study found that motor symptoms and medication explained only $10-30 \%$ of the variation of sleep efficiency, percentage of different sleep stages (including REM sleep), and total sleep time. These authors also suggest that other factors, related to changes in the disease process itself, are impacting sleep quality in PD ${ }^{43}$.
The fact that PD is perceived as a network disorder is contributing to the complexity of this field. The clinical effects of STN-DBS has been suggested to result from modulations of the connectivity within the cortico-striato-thalamo-cortical loop ${ }^{44}$ and of basal ganglia circuits that also affect sleep physiology ${ }^{45}$. Furthermore, the pedunculopontine nucleus and globus pallidus externa are both connected to STN ${ }^{46-48}$ and are important in sleep regulation. Studies have shown that DBS in these areas increases REM sleep ${ }^{46,49}$.

The causes of sleep disturbances thus seem complex and likely multi-factorial. Motor and non-motor PD symptoms, medication, dysregulation of sleep-wake function, and co-morbidities such as sleep-related breathing disorders can probably all play a role $\mathrm{e}^{50-52}$. Therefore, the improvement of sleep quality probably also can be attributed to several mechanisms.

In this study, it was evident that both overall sleep quality and motor-associated sleep symptoms improved significantly. Sleep onset and maintenance insomnia improved less convincingly. Regression analyses did not reveal preoperative variables that predicted the PDSS reduction at 1 year or covariates to the PDSS improvement. Together, this may indicate that STN-DBS causes improvement in sleep disturbances through several mechanisms such as improvement in both nocturnal motor and NMS. There might also be a more direct effect on sleep-wake regulation centers.

Regarding autonomic symptoms, the significant improvement of Scopa-Aut total score at 3 months was no longer evident after 12 months of STN-DBS. However, thermoregulatory function remained significantly improved, due to reduced excessive sweating both during the day and night. This improvement of sudomotor function has been described in several previous publications ${ }^{53}$, and our results further confirm this.

Scopa-Aut total score has been reported to increase by age and disease duration ${ }^{17}$. In a study of 131 patients, Scopa-Aut scores worsened by $20 \%$ after 12 months of follow-up, and the worsening of autonomic symptoms correlated with reduced performance of daily activities of living and health-related quality of life ${ }^{54}$. Thus our findings of lower Scopa-Aut score at 12 months compared to the preoperative score (although not statistically significant) could still imply some degree of long-term improvement when accounting for the expected gradual worsening over time. Few previous reports have used Scopa-Aut to evaluate the effect of STN-DBS on autonomic symptoms. However, one study of 24 patients reported similar results as presented here, where Scopa-Aut initially improved but with subsequent deterioration ${ }^{55}$. Many studies focusing on NMS have used NMSS or NMSQ ${ }^{56,57}$, but these scales reflect total NMS burden and fluctuations and not autonomic dysfunction specifically. However, many of these studies show the same pattern of initial improvement of NMS with later worsening over time ${ }^{23,25}$.

We could not identify any preoperative factors that predicted the change in Scopa-Aut from preoperative to 12 months, but the parallel improvement of PDQ-39 was identified as a significant covariate. This might imply that improvements in autonomic symptoms contribute importantly to improved quality of life.

To our knowledge, this is the only long-term study that combines a relatively high number of patients, with specific evaluations of autonomic and sleep symptoms in PD patients after STN-DBS. The strengths of our study are the prospective design with systematic follow-up, the larger number of patients, and the longer follow-up time compared to previous studies. One limitation of our study is that the presented data were not planned as secondary outcomes but as preplanned investigations in a randomized study with motor symptoms and quality of life as primary endpoints. Thus, for the main variables presented in this article (PDSS and Scopa-Aut), the power to detect statistical differences between the two randomization groups of the parent study was not calculated. No such differences were found, and 
therefore we studied longitudinal changes of these variables in the whole study population. Multiple testing has been performed, but we have tried to correct for this using Bonferroni correction. No control group was included.

In summary, our study confirms a significant and lasting benefit of STN-DBS on sleep quality in PD patients with motor fluctuations. Some aspects of autonomic dysfunction also improved, although only thermoregulatory (sudomotor) function remained significantly improved at 12 months. We could not identify preoperative factors that predicted improvement in PDSS or Scopa-Aut. We did, however, find a close relationship between improved Scopa-Aut and improved quality of life at 1 year. Quality of sleep and autonomic symptoms deserve more focus both in the preoperative and postoperative evaluation of PD patients for STN-DBS. Future studies should consider including these factors among the main outcomes, especially in studies focusing on optimal electrode location, closed-loop DBS, and when exploring new targets.

\section{METHODS}

From 2009 to 2013, 60 patients referred for STN-DBS to the Department of Neurology, Oslo University Hospital were included in a single-center, prospective, randomized, double-blind study. We compared two methods of using up to five trajectories of preoperative microelectrode recordings (MER): single sequential versus multiple simultaneous introduction of MER to guide the placement of the permanent electrode. The detailed description of the study design (including surgical procedure) and the main results of this study on motor and quality of life outcomes have been published previously ${ }^{29}$. The primary endpoints of the parent trial were the differences in motor outcome (scores of MDS-UPDRS III medication-off) and quality of life (PDQ-39), from baseline to 1 year of STN-DBS. The multiple simultaneous MER group had a significantly greater improvement both in MDS-UPDRS III off score and in two PDQ-39 domains (activities of daily living and bodily discomfort) ${ }^{29}$.

Inclusion criteria for surgery were levodopa-responsive PD, with motor fluctuations including dyskinesia or medication-resistant tremor and/or intolerable side effects of dopaminergic drugs. Before inclusion, a neurologist, a psychiatrist, and a neuropsychologist evaluated all patients. Exclusion criteria included previous surgery for PD, marked axial motor symptoms, unresponsiveness to levodopa, significant cognitive impairment, major psychiatric disorders, or significant abnormalities on neuroimaging. All patients signed written informed consent. We have complied with all relevant ethical regulations, and the regional ethics committee (REK) approved the study.

\section{Neurologic evaluations}

Patients were investigated with the MDS-UPDRS to assess non-motor experiences of daily living (Part I), motor experiences of daily living (Part II), motor examination (Part III), and the severity and impact of motor fluctuations (Part IV) ${ }^{58}$. The MDS-UPDRS III (range 0-132) was scored after overnight withdrawal of dopaminergic drugs (medication-off) and after a levodopa dose approximately 1.5 times the patient's usual morning dose (medication-on). Postoperative evaluations were always made in the stimulation-on state. The H\&Y score (0-5) was performed according to the recommendations of the MDS task force ${ }^{59}$. LEDD were calculated as advised by Tomlinson et al. ${ }^{30}$. Disease-specific health-related quality of life was assessed with PDQ-3960,61.

As part of the preplanned investigations performed at baseline, and after 3 and 12 months of STN-DBS, sleep disturbances were assessed with the PDSS and autonomic symptoms with the Scopa-Aut questionnaire. PDSS is a self-rated scale designed to measure common nocturnal problems, sleep disturbances, and excessive daytime sleepiness over the previous week ${ }^{62}$. It consists of 15 items to be scored from 0 (symptom severe and always present) to 10 (symptom-free), and maximum score is 150 (patient is free of all symptoms). Cutoff values of $\leq 83$ for severe sleep abnormalities and $\leq 120$ to detect sleep disturbances have been proposed $^{31,63}$. The Scopa-Aut is a self-administered questionnaire, consisting of 26 items. It assesses the following domains: gastrointestinal symptoms ( 7 items), urinary symptoms (6 items), cardiovascular symptoms ( 3 items), thermoregulation ( 4 items), pupillomotor function ( 1 item), and sexual function ( 2 separate items for each gender) ${ }^{64}$. Each item is scored from 0 (never) to 3 (often), except for question 26 , which is a yes/no question, and consequently not included in our statistical analysis. Total score ranges from 0 to 69 (for both genders), with higher scores expressing more severe symptoms.

\section{Statistical analysis}

Because there were no significant differences in the changes of PDSS or Scopa-Aut from preoperative to 3 months and 12 months between the two randomization groups (independent sample $t$ tests), we analyzed the two groups as one sample for the purpose of this paper.

The sample was assessed with tests for normality. Except for MDSUPDRS IV at 3 and 12 months, variables were normally distributed. Oneway repeated-measures ANOVA were performed to determine the difference between the preoperative and postoperative scores. For MDSUPDRS IV, the Friedman Test was performed. Bonferroni corrections were made for PDSS with denominator 9 (significance level $p<0.006$ ) and for Scopa-Aut denominator 7 (significance level $p<0.007$ ). Where significant $p$ values were found, pairwise comparisons were also performed to see if the significant change was for both time points. In SPSS, this is done in the same model and based on estimated marginal means. These $p$ values are also Bonferroni corrected by multiplying for the three tests $(p<0.02)$.

We defined three subgroups of PDSS severity (PDSS $\leq 83$ (indicating severe sleep abnormality), PDSS 84-120 (detecting sleep disturbances), and PDSS $\geq 121$ (no sleep disturbance detected) according to proposed cutoff values. McNemar's test was performed to compare whether the proportion of patients in these subgroups changed from preoperative to 3 months and from preoperative to 12 months.

Pearson's rank order correlation was performed to compare scores of PDSS, Scopa-Aut, PDQ-39, MDS-UPDRS (I, II, III both medication-off and medication-on, VI), H\&Y, LEDD, age at surgery, and duration of PD for both preoperative and 12-month follow-up scores and for the changes of scores between these time points. For correlation with MDS-UPDRS IV, we also used Spearman's rank correlation, which showed similar correlation coefficients and $p$ levels.

We performed multivariable linear regression analysis to assess (1) which preoperative factors might predict the changes in PDSS and Scopa-Aut from preoperative to 12 months of STN-DBS (prediction models) and (2) whether changes in other selected variables during this treatment period were associated with the changes observed in PDSS and Scopa-Aut scores (covariance models). The variables entered in the models were chosen based on clinical experience and knowledge, hypotheses presented in the literature on variables that can affect improvement in sleep and autonomic symptoms, and through exploratory correlation analyses. We included all variables that showed significant correlation $(\leq 0.05,2$-tailed). We used tolerance $(>0.10)$ and variance inflation factor $(<10)$ to assess for multicollinearity and checked that correlation was $<0.7$. We used a forced entry method, which means that all predictor variables are tested in one block to assess their predictive ability while controlling for the effects of other predictors in the model. The model fit is presented by adjusted $R$ square.

Missing single-item data points for PDSS, Scopa-Aut, and PDQ-39 $(<1 \%$ of total items) were imputed using the last-observation carried-forward method. If the entire questionnaire was missing, these patients were excluded from the analysis. All statistical analyses were performed using IBM SPSS.22.

\section{Reporting summary}

Further information on research design is available in the Nature Research Reporting Summary linked to this article.

\section{DATA AVAILABILITY}

The data that support the findings of this study are not publicly available due to restrictions by Norwegian data protection regulations. The data will be available from the corresponding author upon reasonable request.

Received: 5 July 2019; Accepted: 2 September 2020; Published online: 14 October 2020

\section{REFERENCES}

1. Postuma, R. B. et al. MDS clinical diagnostic criteria for Parkinson's disease. Mov. Disord. 30, 1591-1601 (2015). 
2. Sauerbier, A., Qamar, M. A., Rajah, T. \& Chaudhuri, K. R. New concepts in the pathogenesis and presentation of Parkinson's disease. Clin. Med. 16, 365-370 (2016).

3. Stern, M. B., Lang, A. \& Poewe, W. Toward a redefinition of Parkinson's disease. Mov. Disord. 27, 54-60 (2012).

4. Chaudhuri, K. R., Healy, D. G. \& Schapira, A. H. Non-motor symptoms of Parkinson's disease: diagnosis and management. Lancet Neurol. 5, 235-245 (2006).

5. Lim, S. Y. \& Lang, A. E. The nonmotor symptoms of Parkinson's disease-an overview. Mov. Disord. 25(Suppl 1), S123-S130 (2010).

6. Barone, P. et al. The PRIAMO study: a multicenter assessment of nonmotor symptoms and their impact on quality of life in Parkinson's disease. Mov. Disord. 24, 1641-1649 (2009).

7. Martinez-Martin, P., Rodriguez-Blazquez, C., Kurtis, M. M., Chaudhuri, K. R. \& Group, N. V. The impact of non-motor symptoms on health-related quality of life of patients with Parkinson's disease. Mov. Disord. 26, 399-406 (2011).

8. Martinez-Martin, P. et al. Relationship between the MDS-UPDRS domains and the health-related quality of life of Parkinson's disease patients. Eur. J. Neurol. 21, 519-524 (2014).

9. Hinnell, C. et al. Nonmotor versus motor symptoms: how much do they matter to health status in Parkinson's disease? Mov. Disord. 27, 236-241 (2012).

10. Muller, B., Assmus, J., Herlofson, K., Larsen, J. P. \& Tysnes, O. B. Importance of motor vs. non-motor symptoms for health-related quality of life in early Parkinson's disease. Parkinsonism Relat. Disord. 19, 1027-1032 (2013).

11. Kleiner-Fisman, G. et al. Subthalamic nucleus deep brain stimulation: summary and meta-analysis of outcomes. Mov. Disord. 21(Suppl 14), S290-S304 (2006)

12. Deuschl, G. et al. A randomized trial of deep-brain stimulation for Parkinson's disease. N. Engl. J. Med. 355, 896-908 (2006).

13. Schuepbach, W. M. et al. Neurostimulation for Parkinson's disease with early motor complications. N. Engl. J. Med. 368, 610-622 (2013).

14. Fasano, A., Daniele, A. \& Albanese, A. Treatment of motor and non-motor features of Parkinson's disease with deep brain stimulation. Lancet Neurol. 11, 429-442 (2012).

15. Kim, H. J., Jeon, B. S. \& Paek, S. H. Nonmotor symptoms and subthalamic deep brain stimulation in Parkinson's disease. J. Mov. Disord. 8, 83-91 (2015).

16. Kurtis, M. M., Rajah, T., Delgado, L. F. \& Dafsari, H. S. The effect of deep brain stimulation on the non-motor symptoms of Parkinson's disease: a critical review of the current evidence. NPJ Parkinsons Dis. 3, 16024 (2017).

17. Arnao, V. et al. In patient's with Parkinson disease, autonomic symptoms are frequent and associated with other non-motor symptoms. Clin. Auton. Res. 25, 301-307 (2015).

18. Borgohain, R., Kandadai, R. M., Jabeen, A. \& Kannikannan, M. A. Nonmotor outcomes in Parkinson's disease: is deep brain stimulation better than dopamine replacement therapy? Ther. Adv. Neurol. Disord. 5, 23-41 (2012).

19. Ludwig, J. et al. Effects of subthalamic nucleus stimulation and levodopa on the autonomic nervous system in Parkinson's disease. J. Neurol. Neurosurg. Psychiatry 78, 742-745 (2007).

20. Lilleeng, B., Gjerstad, M., Baardsen, R., Dalen, I. \& Larsen, J. P. The long-term development of non-motor problems after STN-DBS. Acta Neurol. Scand. 132, 251-258 (2015)

21. Klingelhoefer, L., Samuel, M., Chaudhuri, K. R. \& Ashkan, K. An update of the impact of deep brain stimulation on non motor symptoms in Parkinson's disease. J. Parkinsons Dis. 4, 289-300 (2014).

22. Dafsari, H. S. et al. Beneficial effects of bilateral subthalamic stimulation on nonmotor symptoms in Parkinson's disease. Brain Stimul. 9, 78-85 (2016).

23. Dafsari, H. S. et al. Nonmotor symptoms evolution during 24 months of bilateral subthalamic stimulation in Parkinson's disease. Mov. Disord. 33, 421-430 (2018).

24. Witjas, T. et al. Effects of chronic subthalamic stimulation on nonmotor fluctuations in Parkinson's disease. Mov. Disord. 22, 1729-1734 (2007).

25. Ortega-Cubero, S. et al. Effect of deep brain stimulation of the subthalamic nucleus on non-motor fluctuations in Parkinson's disease: two-years' follow-up. Parkinsonism Relat. Disord. 19, 543-547 (2013).

26. Lees, A. J., Blackburn, N. A. \& Campbell, V. L. The nighttime problems of Parkinson's disease. Clin. Neuropharmacol. 11, 512-519 (1988).

27. Tandberg, E., Larsen, J. P. \& Karlsen, K. A community-based study of sleep disorders in patients with Parkinson's disease. Mov. Disord. 13, 895-899 (1998).

28. Gallagher, D. A., Lees, A. J. \& Schrag, A. What are the most important nonmotor symptoms in patients with Parkinson's disease and are we missing them? Mov. Disord. 25, 2493-2500 (2010).

29. Bjerknes, S. et al. Multiple microelectrode recordings in STN-DBS surgery for Parkinson's disease: a randomized study. Mov. Disord. Clin. Pract. 5, 296-305 (2018).

30. Tomlinson, C. L. et al. Systematic review of levodopa dose equivalency reporting in Parkinson's disease. Mov. Disord. 25, 2649-2653 (2010).

31. Martinez-Martin, P. et al. SCOPA-sleep and PDSS: two scales for assessment of sleep disorder in Parkinson's disease. Mov. Disord. 23, 1681-1688 (2008).
32. Arnulf, I. et al. Improvement of sleep architecture in PD with subthalamic nucleus stimulation. Neurology 55, 1732-1734 (2000)

33. Iranzo, A., Valldeoriola, F., Santamaria, J., Tolosa, E. \& Rumia, J. Sleep symptoms and polysomnographic architecture in advanced Parkinson's disease after chronic bilateral subthalamic stimulation. J. Neurol. Neurosurg. Psychiatry 72, 661-664 (2002)

34. Monaca, C. et al. Effects of bilateral subthalamic stimulation on sleep in Parkinson's disease. J. Neurol. 251, 214-218 (2004).

35. Hogl, B. et al. Scales to assess sleep impairment in Parkinson's disease: critique and recommendations. Mov. Disord. 25, 2704-2716 (2010).

36. Hjort, N., Ostergaard, K. \& Dupont, E. Improvement of sleep quality in patients with advanced Parkinson's disease treated with deep brain stimulation of the subthalamic nucleus. Mov. Disord. 19, 196-199 (2004)

37. Chahine, L. M., Ahmed, A. \& Sun, Z. Effects of STN DBS for Parkinson's disease on restless legs syndrome and other sleep-related measures. Parkinsonism Relat. Disord. 17, 208-211 (2011).

38. Kharkar, S. et al. Changes in Parkinson's disease sleep symptoms and daytime somnolence after bilateral subthalamic deep brain stimulation in Parkinson's disease. NPJ Parkinsons Dis. 4, 16 (2018).

39. Chahine, L. M. et al. Association between dopaminergic medications and nocturnal sleep in early-stage Parkinson's disease. Parkinsonism Relat. Disord. 19, 859-863 (2013)

40. Wailke, S., Herzog, J., Witt, K., Deuschl, G. \& Volkmann, J. Effect of controlledrelease levodopa on the microstructure of sleep in Parkinson's disease. Eur. J. Neurol. 18, 590-596 (2011).

41. Dhawan, V. et al. The range and nature of sleep dysfunction in untreated Parkinson's disease (PD). A comparative controlled clinical study using the Parkinson's Disease Sleep Scale and selective polysomnography. J. Neurol. Sci. 248, 158-162 (2006).

42. Odekerken, V. J. et al. Subthalamic nucleus versus globus pallidus bilateral deep brain stimulation for advanced Parkinson's disease (NSTAPS study): a randomised controlled trial. Lancet Neurol. 12, 37-44 (2013).

43. Martinez-Ramirez, D. et al. A polysomnographic study of Parkinson's disease sleep architecture. Parkinsons Dis. 2015, 570375 (2015).

44. Mueller, K. et al. Brain connectivity changes when comparing effects of subthalamic deep brain stimulation with levodopa treatment in Parkinson's disease. Neuroimage Clin. 19, 1025-1035 (2018).

45. Sharma, V. D., Sengupta, S., Chitnis, S. \& Amara, A. W. Deep brain stimulation and sleep-wake disturbances in parkinson disease: a review. Front. Neurol. 9, 697 (2018).

46. Thevathasan, W. \& Moro, E. What is the therapeutic mechanism of pedunculopontine nucleus stimulation in Parkinson's disease? Neurobiol. Dis. https://doi.org/ 10.1016/j.nbd.2018.06.014 (2018).

47. Nowacki, A. et al. Pedunculopontine nucleus: an integrative view with implications on deep brain stimulation. Neurobiol. Dis. https://doi.org/10.1016/j. nbd.2018.08.015 (2018).

48. Stefani, A., Grandi, L. C. \& Galati, S. Deep brain stimulation of the pedunculopontine nucleus modulates subthalamic pathological oscillations. Neurobiol. Dis. https://doi.org/10.1016/j.nbd.2018.11.006 (2018).

49. Qiu, M. H., Chen, M. C., Wu, J., Nelson, D. \& Lu, J. Deep brain stimulation in the globus pallidus externa promotes sleep. Neuroscience 322, 115-120 (2016).

50. Sixel-Doring, F., Trautmann, E., Mollenhauer, B. \& Trenkwalder, C. Age, drugs, or disease: what alters the macrostructure of sleep in Parkinson's disease? Sleep Med. 13, 1178-1183 (2012).

51. Amara, A. W., Chahine, L. M. \& Videnovic, A. Treatment of sleep dysfunction in Parkinson's disease. Curr. Treat. Options Neurol. 19, 26 (2017).

52. Albers, J. A., Chand, P. \& Anch, A. M. Multifactorial sleep disturbance in Parkinson's disease. Sleep Med. 35, 41-48 (2017).

53. Basiago, A. \& Binder, D. K. Effects of deep brain stimulation on autonomic function. Brain Sci. 6, 33 (2016).

54. Merola, A. et al. Autonomic dysfunction in Parkinson's disease: a prospective cohort study. Mov. Disord. 33, 391-397 (2018).

55. Kurcova, S. et al. Bilateral subthalamic deep brain stimulation initial impact on nonmotor and motor symptoms in Parkinson's disease: an open prospective single institution study. Medicine 97, e9750 (2018).

56. Chaudhuri, K. R. et al. International multicenter pilot study of the first comprehensive self-completed nonmotor symptoms questionnaire for Parkinson's disease: the NMSQuest study. Mov. Disord. 21, 916-923 (2006).

57. Chaudhuri, K. R. et al. The metric properties of a novel non-motor symptoms scale for Parkinson's disease: results from an international pilot study. Mov. Disord. 22, 1901-1911 (2007).

58. Goetz, C. G. et al. Movement Disorder Society-sponsored revision of the Unified Parkinson's Disease Rating Scale (MDS-UPDRS): scale presentation and clinimetric testing results. Mov. Disord. 23, 2129-2170 (2008). 
59. Goetz, C. G. et al. Movement Disorder Society Task Force report on the Hoehn and Yahr staging scale: status and recommendations. Mov. Disord. 19, 1020-1028 (2004).

60. Jenkinson, C., Fitzpatrick, R., Peto, V., Greenhall, R. \& Hyman, N. The Parkinson's Disease Questionnaire (PDQ-39): development and validation of a Parkinson's disease summary index score. Age Ageing 26, 353-357 (1997).

61. Peto, V., Jenkinson, C., Fitzpatrick, R. \& Greenhall, R. The development and validation of a short measure of functioning and well being for individuals with Parkinson's disease. Qual. Life Res. 4, 241-248 (1995).

62. Chaudhuri, K. R. et al. The Parkinson's disease sleep scale: a new instrument for assessing sleep and nocturnal disability in Parkinson's disease. J. Neurol. Neurosurg. Psychiatry 73, 629-635 (2002).

63. Uemura, Y. et al. Validation of the Parkinson's disease sleep scale in Japanese patients: a comparison study using the Pittsburgh Sleep Quality Index, the Epworth Sleepiness Scale and Polysomnography. J. Neurol. Sci. 287, 36-40 (2009).

64. Visser, M., Marinus, J., Stiggelbout, A. M. \& Van Hilten, J. J. Assessment of autonomic dysfunction in Parkinson's disease: the Scopa-Aut. Mov. Disord. 19, 1306-1312 (2004).

\section{ACKNOWLEDGEMENTS}

The authors thank all study participants. Statistician Are Hugo Pripp has advised and guided the authors on the statistical analysis. The study has been supported by the South-Eastern Norway Regional Health Authority. M.T. is supported by the Research Council of Norway and a Career Fellowship grant from the South-Eastern Norway Regional Health Authority. I.M.S. has during the work with this study been supported by a grant from the Norwegian Research Council and from private donations.

\section{AUTHOR CONTRIBUTIONS}

The study was designed by E.D., I.M.S., and M.T. M.T., I.M.S., and S.B. carried out the clinical assessments of patients. T.J.H. and S.B. performed analyses of the data. S.B. drafted the manuscript. All the co-authors critically revised the manuscript for intellectual content and approved the final version for publication.

\section{COMPETING INTERESTS}

The authors declare no competing interests.

\section{ADDITIONAL INFORMATION}

Supplementary information is available for this paper at https://doi.org/10.1038/ s41531-020-00131-0.

Correspondence and requests for materials should be addressed to S.B.

Reprints and permission information is available at http://www.nature.com/ reprints

Publisher's note Springer Nature remains neutral with regard to jurisdictional claims in published maps and institutional affiliations.

Open Access This article is licensed under a Creative Common Attribution 4.0 International License, which permits use, sharing, adaptation, distribution and reproduction in any medium or format, as long as you give appropriate credit to the original author(s) and the source, provide a link to the Creative Commons license, and indicate if changes were made. The images or other third party material in this article are included in the article's Creative Commons license, unless indicated otherwise in a credit line to the material. If material is not included in the article's Creative Commons license and your intended use is not permitted by statutory regulation or exceeds the permitted use, you will need to obtain permission directly from the copyright holder. To view a copy of this license, visit http://creativecommons. org/licenses/by/4.0/.

(c) The Author(s) 2020 